Original Article

\title{
Clinical effect of pro-biotic containing Bacillus coagulans on plaque induced gingivitis : A randomised clinical pilot study.
}

\author{
Maithri Jagadeesh K. ${ }^{1}$, Nina Shenoy ${ }^{2}$, Avaneendra Talwar ${ }^{3}$, Smitha Shetty ${ }^{4}$ \\ ${ }^{1}$ Former BDSStudent, ${ }^{2}$ Professor, ${ }^{3,4}$ Reader, Department of Periodontics, A.B. Shetty M emorial Institute of Dental Sciences, \\ Nitte University, Mangalore.
}

*Corresponding Author : Nina Shenoy, Professor, Department of Periodontics, A.B. Shetty Memorial Institute of Dental Sciences, Deralakatte, Nitte University, M angalore. E-mail : ninashenoy@gmail.com.

$\begin{array}{ll}\text { Received } & : 22.06 .2017 \\ \text { Review Completed } & : 28.08 .2017 \\ \text { Accepted } & : 30.08 .2017\end{array}$

Keywords : Bacillus coagulans; gingivitis; probiotics

\begin{tabular}{|c|}
\hline Access this article online \\
\hline Quick Response Code \\
\hline
\end{tabular}

\begin{abstract}
:
Objective : To evaluate the clinical effect of Bacillus coagulans on plaque induced gingivitis.

Method : Thirty subjects with plaque induced were enrolled in the study. At baseline, Gingival Index (GI) and Plaque Index (PI) were assessed. Saliva samples were collected for glutathione peroxidise (GPx) activity analysis and to determine load of lactobacilli. Subsequently participants were randomly provided with chewable tablets to be consumed $3 \mathrm{X}$ daily for 3 month containing 100 million colony forming units (CFU)/tablet of B. coagulans or without $B$. coagulans (placebo). After3 months, recording of GI, PI and saliva sampling were repeated.

Result : At baseline, mean GI, and mean PI did not differ significantly between both groups. At re-evaluation, mean $\mathrm{Gl}$, and bleeding on probing of the probiotic group were both significantly $(p<0.0001$ ) lower than in the placebo group. M ean PI level did not differ significantly between the groups. In the probiotic group, mean glutathione peroxide activity (Gpx)was significantly ( $p$ $<0.02$ ) lower than in the placebo group at re-evaluation.
\end{abstract}

Conclusion : The consumption of probiotic containing Bacillus coagulans seems to modulate inflammatory response in plaque induced gingivitis.

\section{Introduction}

Plaque induced gingivitis is a frequent clinical finding, with a prevalence of over $90 \%$ in the general population. Plaque induced gingivitis is reversible, but may progress to periodontitis, in susceptible patients, if left untreated. Professionally administered plaque removal followed by reinstitution of oral hygiene is indispensable in maintaining periodontal health. ${ }^{[1]}$

However, failure to completely resolve gingival inflammation remains a problem as plaque removal may not significantly reverse the overgrowth of sub-gingival flora. It has been hypothesised that residual inflammation following plaque removal, aids the stabilization of subgingival flora composition, even in the absence of plaque or periodontal pockets. The persistence of specific pathogenic phyla even after oral hygiene measures may result in progression of periodontal disease. ${ }^{[2]}$ Hence, in order to enhance the clinical outcome of plaque removal, several adjunctive treatments such as antiseptics, local and systemic antibiotics, photodynamic therapy have been proposed to reduce the microbial flora. However, none of these adjunctive treatments have shown superior efficacy to scaling and root planing alone. ${ }^{[3]}$

Bacterio therapy, is a promising alternative adjunctive treatment method to combat inflammation. Probiotics are one of these new agents. Probiotics are harmless living micro-organisms which, when administered in adequate amounts, confer a health benefit to the host by suppressing/displacing endogenous and exogenous pathogens and by modulating the immune system towards an anti-inflammatory response. ${ }^{[4]}$

To date, a number of studies have evaluated the adjunctive efficacy of probiotics containing different Lactobacillus and Bifidobacterial strains for the treatment of plaque induced 
gingivitis with conventional scaling and root planing, with positive results. In addition, there are other genera of bacteria like Bacillus coagulans that have probiotic effect and may have a positive effect in the management of periodontal disease. ${ }^{[4,5]}$

Bacillus coagulans, earlier known as Lactobacillus sporogenes, is a gram positive, lactic acid producing, and spore forming bacteria, which is non-pathogenic and safe for human consumption. Due its spore forming characteristics these bacteria have the potential to survive industrial processing. It is reported to support a healthy balance of microflora in the intestinal environment. To the investigator's knowledge, there are no controlled studies evaluating their role in the prevention and treatment of gingivitis. ${ }^{[6,7,8]}$

Since, the effects of probiotics are strain-, dosage- and mode of application-dependent, ${ }^{[9]}$ the aim of the present pilot study, was to evaluate the clinical efficacy of probiotic containing Bacillus coagulans in a chewing tablet form in the treatment of plaque induced gingivitis.

\section{Materials and Method}

\section{Study Population}

A double-blind, placebo-controlled, prospective study was designed.(Table I) The study protocol was approved by the institutional ethical committee and written consent was obtained from patients before enrolling them for the study.

Subjects in age group of 18-50 years, diagnosed with gingivitis, based on the gingival index score (Loe and Silness, 1963 ${ }^{[10]}$, in the Department of Periodontics of A. B. Shetty Memorial Institute of Dental Sciences, Deralakatte, Mangalore, were included. Patients were randomly assigned to probiotic group (test) or the placebo group (control), with 15 patients in each group. The second author (NS) was the only person aware of treatment given to the patient's, and coding was not unblinded until the end of the study. The clinical examiner (MJ) and the patients were blinded.

The exclusion criteria were: (i) patients receiving any kind of local or systemic decontamination treatment of the oral cavity in the last 3 months, or periodontal treatment in the last 6 months; (ii) uncontrolled periodontal disease; (iii) patients with systemic disorders capable of influencing the treatment results; (iv) smokers; (v) incomplete protocols due to a lack of patient cooperation; (vi) failure to provide informed consent to participate in the study ; (vii) Patients on antibiotic therapy.;(viii) Subjects using probiotic supplements; $x$ ) Pregnant, lactating and women in their menstrual phase. The inclusion criteria were: (i)) Subjects with minimum complement of 20 teeth; (ii) Systemically healthy subjects with moderate to severe gingivitis.

\section{Probiotic Product}

The study product (probiotic) Bacillus coagulans, which was available as free flowing, greyish white powder (Unique Biotech Limited, Hyderabad, India) was manufactured in the form of chewable tablets at Srinivas College of Pharmacy, Valachil. Each probiotic tablet contained 100 million colony forming units (CFU)/tablet. It was tested against a placebo tablet containing starch ( $150 \mathrm{mg} /$ tablet), identical in appearance with the probiotic. The dose was 3 tablets to be taken orally every day throughout the test period. Subjects were instructed to place the tablet in the oral cavity for few minutes, allowing them to dissolve.

\section{Clinical parameters}

All clinical measurements were obtained from Ram fjord's six teeth $(16,21,24,36,41 \& 44$ in the FDI two-digit notation system) ${ }^{[1]}$ in all subjects at each visit and considered representative of the whole dentition. Bleeding on probing (BOP $)^{[12]}$, Gingival Index $(\mathrm{GI})^{[10]}$ and Plaque Index $(\mathrm{PI})^{[13]}$ were recorded.

The study was performed over a 3week period. Test group subjects and control group subjects were given probiotic tablets and placebo tablets respectively thrice daily for 3 weeks as per the above-mentioned instructions. Subjects were instructed not to change their oral hygiene regimens. No oral hygiene instructions were given under the experimental period. Clinical parameters were recorded and saliva samples were collected from all subjects on days 0 (baseline; BL) and 22 (3 weeks; 3W). 


\section{Collection of Saliva}

Un stimulated whole saliva samples were obtained by expectoration. Subjects were asked to spit approximately $3.0 \mathrm{ml}$ of saliva into sterile bottles. Saliva samples were collected between 09:00 and 12:00 hours. Samples were stored at $4^{\circ} \mathrm{C}$ until use and then delivered to the laboratory for analysis.

\section{Glutathione Peroxidise Activity}

The collected saliva samples $(1 \mathrm{ml})$ were centrifuged at $2000 \mathrm{rpm}$ for 5 minutes and the supernatant was stored in clean Eppendorf tubes in the freezer at $-20^{\circ} \mathrm{C}$ until analysis was done. Glutathione peroxidise activity in the saliva was determined by using UV Spectrophotometer at $412 \mathrm{~nm}$. The enzyme activity was expressed as $\mu \mathrm{g}$ of glutathione (GSH) consumed/min/mg protein.

\section{Microbiological Analysis}

The saliva samples $(1 \mathrm{ml})$ were streaked onto Rogosa $\mathrm{SL}$ HiVeg ${ }^{\mathrm{TM}}$ Agar media and incubated at $37^{\circ} \mathrm{C}$ for 24 to 48 hours. The colonies of lactobacilli were confirmed by Gram staining and colony morphology. The load of lactobacilli in the saliva was determined by microbial assay.

\section{Statistical Analysis}

The obtained data was analysed using unpaired Student's t-test, to compare the before and after values. A p-value of $<0.001$ was considered to be statistically significant (s) and $p$-value of $>0.05$ was considered not significant (ns).

\section{Results}

30 patients were included in the study and randomized in two groups of 15 patients (test or placebo). All patients attended all the programmed visits. Results of the study are summarised in table ll

\section{Clinical outcome}

A significant difference was detected between the groups for BOP $(p<0.0001)$. A statistically significant decrease in $\mathrm{BOP}$ in the probiotic group subjects was observed from baseline till the end of the study $(p<0.0001)$, while no significant difference was found in BOP in the placebo group subjects $(p>0.05)$.
For $\mathrm{Gl}$, a statistically significant decrease was observed in the Probiotic group subjects after the experimental period $(p<0.0001)$, while no statistically significant difference was seen in placebo group subjects after the study period $(p>0.05)$. [Table II]

For PI, no statistically significant difference was observed over the study period in both the Probiotic and Placebo groups $(p>0.05)$.

\section{Microbiological outcomes in saliva samples}

There was no statistically significant difference in the colony count of Lactobacilli in saliva in both the probiotic and placebo groups during the study period. (Data not shown)

\section{Glutathione peroxide (GPx) activity}

The GPx activity in the probiotic group subjects were significantly lower, $(p \varangle 0.0001)$ whereas the GPx activity in the placebo group subjects was not statistically different after the experimental period $(p>0.05)$.

\section{Discussion}

The present double-blind, placebo-controlled, prospective pilot study was designed to study the clinical efficacy and microbial impact of probiotics containing Bacillus coagulans (100 CFU/tablet)in the treatment of moderate to severe plaque induced gingivitis. The concept of probiotic bacterio therapy has been proposed for the treatment of periodontal disease due to their ability to inhibit growth of pathogenic bacteria as well as their antiinflammatory properties. The probiotic tablets in the present study were administered for a 3-week period. ${ }^{[14,15]}$

In the present study effects of probiotic bacteria on the periodontal clinical parameters were evaluated. In the probiotic group a statistically significant reduction in $\mathrm{Gl}$ and BOP was observed (Table II). Our results with respect to GI and BOP agree with previous studies. ${ }^{[16,17]}$ where Lactobacilli reuteri was used. In contrast, Staab B et al., ${ }^{[18]}$ who used Lactobacillus casei as a probiotic, reported no statistically significant change. The anti-inflammatory effect of the probiotic may be due to its antagonist action against pathogens by inhibiting the adhesion and growth of 
Table I : Experimental Design

No. of Subjects : 30

Subjects reporting to the Department of Periodontics

of A.B. Shetty Memorial Institute of Dental

Sciences, were randomly assigned into 2 groups

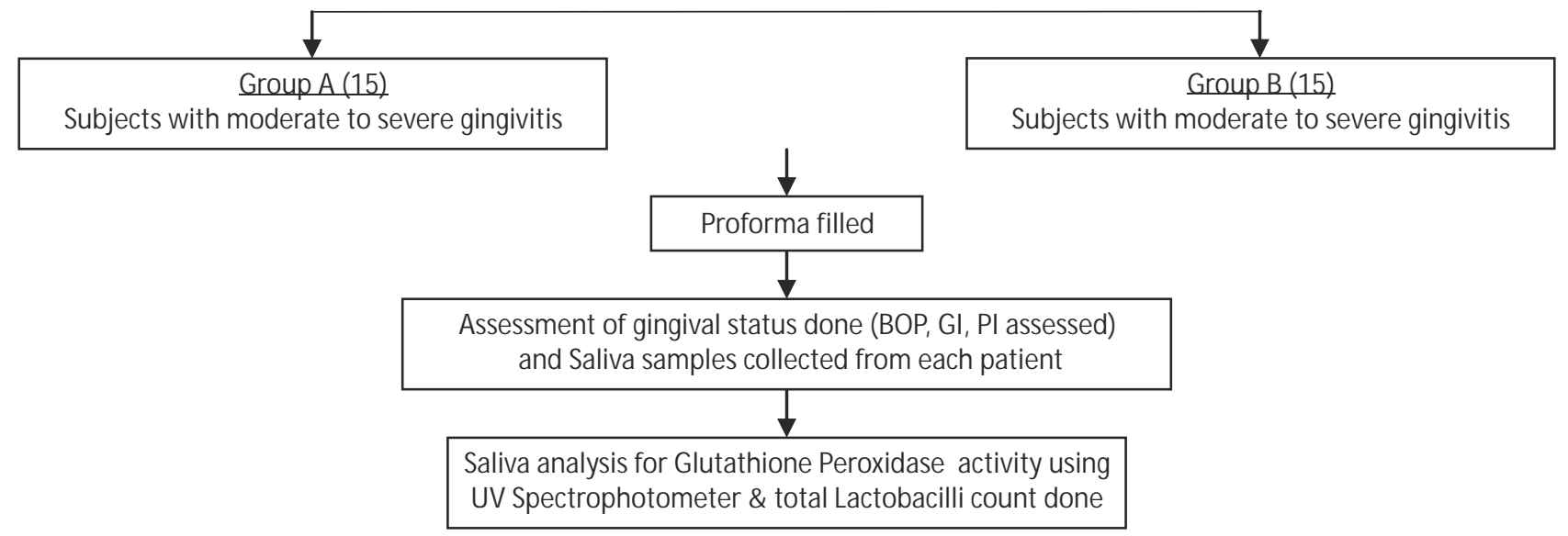

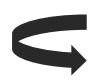

Group A subjects given 3 probiotic tablets / day
Group B subjects given 3 placebo tablets/ day

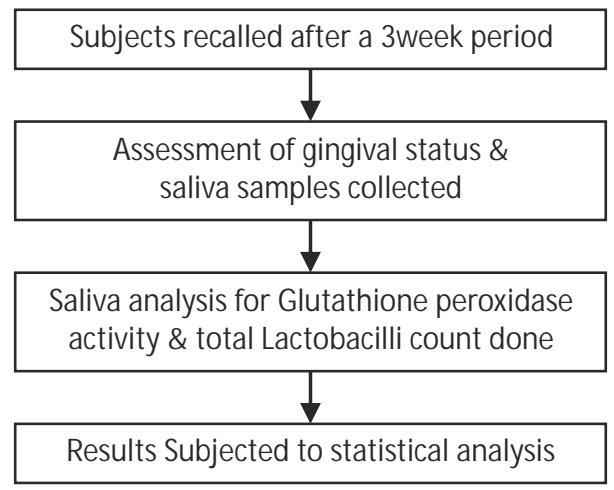

pathogens and also exert an influence on local and systemic immune responses. Furthermore, Bacillus coagulans have been shown to modulate the immune system by production of short chain fatty acids such as butyric acid and stimulating the production of IL-10. ${ }^{[7,8,15]}$

In order to determine the inflammatory status of the gingival, glutathione peroxidise activity was evaluated. Glutathione peroxidise is an antioxidant (AO) that catalyses the breakdown of reactive oxygen species (ROS). In health, the balance is maintained among oxidants and antioxidants. Under pathological conditions such as chronic inflammation, a disturbance in favour of ROS production results in oxidative stress. ${ }^{[19]}$ Whole saliva was chosen as the bio fluid to evaluate the levels of Glutathione peroxidise, as it contains gingival crevicular fluid, immune cells, and tissue metabolites and reflects most closely the predominant intra-oral condition. 
Table II : Analysis of Gingival Index (GI) scores, Plaque Index (PII) scores, bleeding on probing and Glutathione peroxidise (GPx) activity

\begin{tabular}{|c|c|c|}
\hline Probiotic Group & $\begin{array}{c}\text { Placebo Group } \\
n=15\end{array}$ & $\begin{array}{c}P \\
n=15\end{array}$ \\
\hline & Mean \pm SD & Mean \pm SD \\
\hline GI baseline & $1.6 \pm 0.8$ & $1.6 \pm 0.2 \mathrm{~ns}^{*}$ \\
\hline GI re-evaluation & $1.5 \pm 0.1$ & $1.6 \pm 0.3 \mathrm{~s} * *$ \\
\hline$P$-value $<0.0001 * *$ & $>0.05 \mathrm{~ns}^{*}$ & \\
\hline PI baseline & $1.5 \pm 0.6$ & $1.4 \pm 0.8 \mathrm{~ns}^{*}$ \\
\hline PI re-evaluation & $1.5 \pm 0.2$ & $1.4 \pm 0.7 \mathrm{~ns}$ \\
\hline P-value $>0.05 \mathrm{~ns}$ & $>0.05 \mathrm{~ns}$ & \\
\hline BOP baseline & $81.3 \pm 12.6$ & $80.5 \pm 20.8 n^{*}$ \\
\hline BOP re-evaluation & $75.89 \pm 11.2$ & $79.3 \pm 21.2 \mathrm{~s}^{* *}$ \\
\hline P-value $<0.0001 \mathrm{~s}^{* *}$ & $>0.05 \mathrm{~ns}^{*}$ & \\
\hline Gpx activity baseline (pg/ ml) & $132.9 \pm 21.9$ & $131.0 \pm 24.9$ \\
\hline GPx activity re-evaluation (pg/ ml) & l) $89.7 \pm 15.5$ & $131.6 \pm 24.6$ \\
\hline \begin{tabular}{l|l}
$P$-value $<0.0001 \mathrm{~s}^{* *}$ & \\
\end{tabular} & $>0.05 \mathrm{~ns}^{*}$ & \\
\hline
\end{tabular}

In this study, salivary glutathione peroxide activity (GPx) indicated a statistically significant decrease $(P<.0001)$ (Table II) following 3 weeks of probiotic therapy in the probiotic group. Similar findings were reported earlier, where it was demonstrated that salivary glutathione peroxide activity increases in subjects diagnosed with plaque induced gingivitis as compared to healthy subjects and is reduced post therapy. ${ }^{[20]}$ The findings observed can be explained as follows; during the initial stages of inflammation, GPxactivity is increased to compensate for the increased production of ROS. Following treatment with probiotics, reduction in inflammation was reflected in reduced GPxactivity.

In order to determine the influence of probiotics on beneficial oral bacteria, salivary levels of lactobacilli were evaluated. The salivary level of lactobacilli was fairly stable with no statistically significant alterations found between the groups. This indicated that the probiotic has little effect

\section{References}

1. Needleman I, Suvan J, Moles DR, Pimlott J. A systematic review of professional mechanical plaque removal for prevention of periodontal diseases. J ClinPeriodontol 2005; 32 (Suppl. 6): 229-282.

2. Paes Batista da Silva, Moss K, Preisser J, Marchesan JT, Ward M, Offenbacher S. Microbial profiling in experimentally induced biofilm overgrowth among patients with various periodontal states. Periodontol. 2016Jan;87(1):27-35.

3. Takasaki AA, Aoki A, M izutani K, Frank Schwarz F, Sculean A, Wang CY, Koshy G, Romanos G, Ishikawa I\& Izumi Y.Application of antimicrobia on beneficial bacteria. This result was in agreement with an earlier report, ${ }^{[2]]}$ where Lactobacillus reuteri in chewing gum wasused.

This study has several limitations such as the following, it was a short term pilot study involving a small patient population, no treatment group was included and only patients with gingivitis were included.

Within the limitations of this study, administration of probiotics containing Bacillus coagulans, demonstrated improvement in the inflammatory status of the gingiva but had minimal effect on Lactobacilli count. In addition, the glutathione peroxidise level was significantly reduced in the probiotic group indicating, a probable beneficial effect of Bacillus coagulans on periodontal health. Further studies involving a larger population and over longer periods are required to confirm these results.

\section{Conclusion}

The need to promote health in a natural way has led to increased research on probiotics. Probiotics contain beneficial bacteria which have proven to improve the immunity and absorption of essential vitamins and elements necessary for maintenance of health.

In this pilot study, the probiotic Bacillus coagulans was used in a chewable form in chronic gingivitis patients over a 3 week period during which, there was a significant improvement in the gingival health and noted reduction in glutathione peroxidise activity in the probiotic group. Hence, within the limitations of this study it can be concluded that probiotics can be used as adjuncts to nonsurgical periodontal therapy to improve and maintain oral health.

photodynamic therapy in periodontal and peri-implant diseases Periodontol 20002009:51:109-140.

4. Teughels W, Durukan A, Ozcelik O, Pauwels M, Quirynen M \&Haytac MC. Clinical and microbiological effects of Lactobacillus reuteri probiotics in the treatment of chronic periodontitis: a randomized placebo-controlled study. I ClinPeriodontol2013;40: 1025-35.

5. Hallstrom H, Lindgren S, Yucel-Lindberg T, Dahlen G, Renvert $S$, Twetman S. Effect of probiotic lozenges on inflammatory reactions and oral biofilm during experimental gingivitis. ActaOdontolScand2013; 
71:828-833.

6. Bora PSP, Puri V, Bansal AK. Physicochemical Properties and Excipient Compatibility studies of Probiotic Bacillus coagulans Spores. Sci Pharm. 2009; 77; 625-637)

7. Nyangale EP, Farmer S, Cash HA, et al. Bacillus coagulans GBI-30, 6086 Modulates Faecalibacteriumprausnitzii in Older Men and Women. J Nutr. 2015;145(7):1446-1452.)

8. Fitzpatrick LR. Probiotics for the treatment of Clostridium difficile associated disease. World Journal of Gastrointestinal Pathophysiology 2013; 4(3): 47-52.

9. Teughels W, Loozen G, Quirynen M. Do probiotics offer opportunities to manipulate the periodontal oral microbiota? J ClinPeriodontol 2011;38(suppl 11): 159-177.

10. Löe $H$ \&Silness J. Periodontal disease in pregnancy. ActaOdontologicaScandinavica 1963; 21:533-551.

11. Ramfjord S.P. Indices for prevalence and incidence of periodontal disease.J Periodontol 1959; 30: 51-59.

12. Ainamo J \& Bay l. Problems and proposals for recording gingivitis and plaque. International Dental J ournal 1975; 25: 229-235.

13. Silness J \&Löe H. Periodontal disease in pregnancy II. Correlation between oral hygiene and periodontal condition. ActaOdontologicaScandinavica 1964; 24: 747-759.

14. Teughels, W., Essche, M., Sliepen, I. \&Quirynen, M. Probiotics and oral healthcare. Periodontology 2000 2008;48: 111- 147.
15. Haukioja A. Probiotics and Oral Health. European Journal of Dentistry. 2010 July; 4(3); 348-355.

16. Krasse P, Carlsson B, Dahl C, Paulsson A, Nilsson A, Sinkiewicz G. Decreased gum bleeding and reduced gingivitis by the probiotic Lactobacillus reuteri. Swedish Dental Journal. 2006; 30(2): 55-60.

17.Shimauchi H, M ayanagi G, Nakaya S, M inamibuchi M, Ito Y, Yamaki K, Hirata H. Improvement of periodontal condition by probiotics with Lactobacillus salivarius WB21: a randomized, double-blind, placebocontrolled study.J ClinPeriodontol 2008; 35: 897-905.

18. Staab B, Eick S, Knöfler G, Jentsch H. The influence of a probiotic milk drink on the development of gingivitis: a pilot study. J ClinPeriodontol 2009; 36(10): 850-856.

19. Baltacioglu E, Akalin FA, Alver A, Balaban F, U"nsal M , Karabulut E. Total antioxidant capacity and superoxide dismutase activity levels in serum and gingival crevicular fluid in post-menopausal women with chronic periodontitis. J ClinPeriodontol 2006; 33: 385-392.

20. Buduneli N, KardeSler L, ISik H, Willis CS, Hawkins SI, Kinane DF, Scott DA. Effects of smoking and gingival inflammation on salivary antioxidant capacity. J ClinPeriodontol 2006; 33: 159-164.

21. Caglar E, Kavaloglu SC, Kuscu OO, Sandalli N, Holgerson PL, Twetman S. Effect of chewing gums containing xylitol or probiotic bacteria on salivary mutans streptococci and Lactobacilli. Clin Oral Investig2007; 11(4):425-429. 